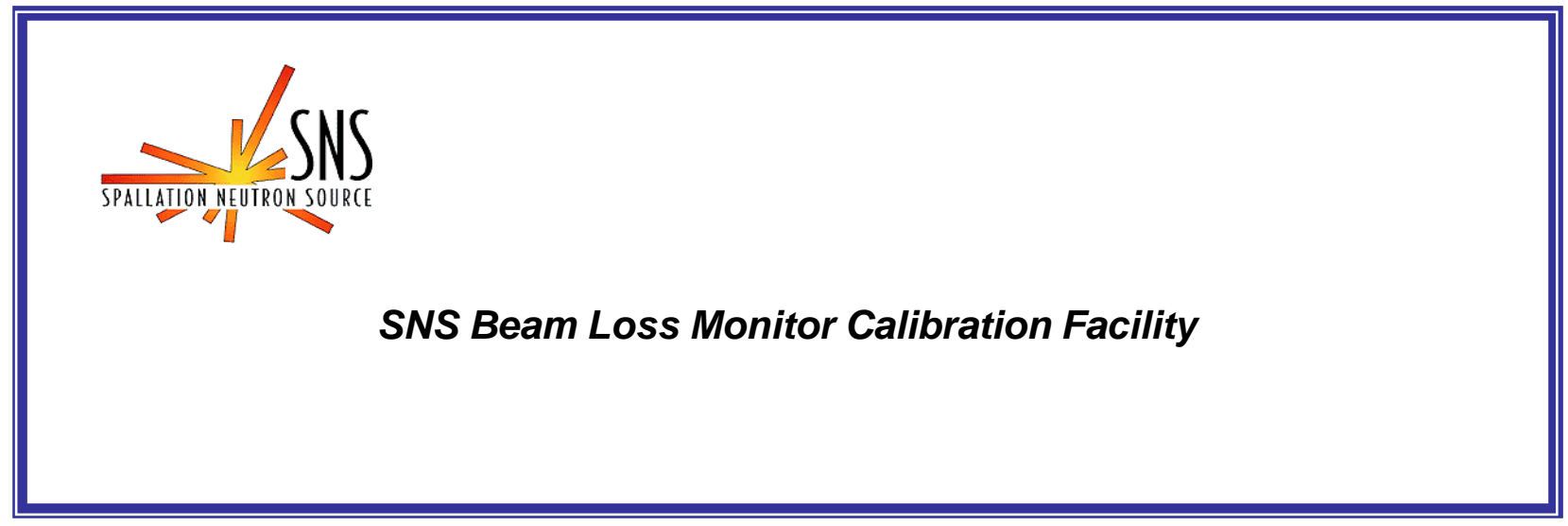

BNL/SNS TECHNICAL NOTE

NO. 125

R.L. Witkover, D. Gassner, Y. Leng

TechSource, Inc., Santa Fe, NM

BNL, Upton, NY 11973, USA

August 27, 2003

COLLIDER-ACCELERATOR DEPARTMENT

BROOKHAVEN NATIONAL LABORATORY

UPTON, NEW YORK 11973 


\title{
SNS Beam Loss Monitor Calibration Facility
}

\author{
R. L. Witkover†, D. Gassner*, Y. Leng* \\ $†$ TechSource, Inc., Santa Fe, NM \\ *Brookhaven National Laboratory, Upton, NY
}

\section{Background}

The Ion Chamber Test facility originally developed for calibrating RHIC Beam Loss Monitors (BLMs) has been upgraded to allow testing of the new SNS ion chambers and to incorporate improved electronics. The Test Facility is located in the rear of Building 922 in the sunken pit area. Details of the new facility are described. The original Ion Chamber Test Stand was constructed to test the ion chambers for RHIC. These are based on a design in 1982 by Shafer ${ }^{1}$ for the Tevatron. Its packaging was modified for RHIC but retained the original glass detector ${ }^{2}$. This detector had an active volume defined by a an outer cylindrical electrode 4-inches long and 1.5-inches diameter, filled with Argon at $725 \mathrm{~mm} \mathrm{Hg}$. With an inner electrode diameter of 0.25 -inches, the design had a long ion collection time $(\sim 700 \mu \mathrm{sec}$ at $2000 \mathrm{~V})$ although the electrons were collected in microseconds. To significantly improve the ion collection time and reduce saturation effects a new ion chamber was designed with a larger inner diameter and greater length to give the same active volume and calibration ${ }^{3}$. Other design changes needed to allow the higher voltage gradient resulted in a new design in stainless steel and ceramic. The physical configuration of the original test stand had to be modified to allow for the greater active length.

The electronics used to condition the signal and cycle through the voltage range was developed in the pre-RHIC era. The output of an in-house design gated integrator was read by a digital scope under LabView control. Bias voltage was provided by a high voltage power supply was controlled by an digital-to-analog output module via LabView. Since the signal levels were tens of pico-Amps considerable care was required to obtain usable signals. Averaging over many readings was required. Over the years the noise environment in the lab became difficult as new projects occupied the building. This and aging of the equipment caused the quality of the data to deteriorate. It was decided to replace the electronics with commercial units with IEEE-488 interfaces.

\section{Mechanical Changes to the Test Stand}

A mechanical fixture was built to fix the positions of the source and ion chamber under test. A phenolic board with rectangular strips defined a box for the location of the radiation source container ("Pig") and a "tee" stand mount fixed the location of the ion chamber. The top of the tee-stand was designed to accept the RHIC ion chamber housing and was positioned $20 \mathrm{~cm}$ from the center of the radiation source. This distance was selected after measurements showed the field to be sufficiently uniform over the active volume at this distance. With the new chamber's greater length, a new tee stand had to be designed and positioned further back. 


\section{Determination of the New Location}

The radiation source is a Cesium-137 gamma emitter $(662 \mathrm{KeV})$ which had an activity of 158.1 milli-Curies on July 1, 1997. The pig containing the source had a conical opening of approximately 30 degrees, with 3 attenuating shutters. Measurements were made to determine the radiation field uniformity using a Teletector Model 6112. The results are shown below. The asymmetry at 16 and $26 \mathrm{~cm}$ is due to the shutters which were subsequently removed.

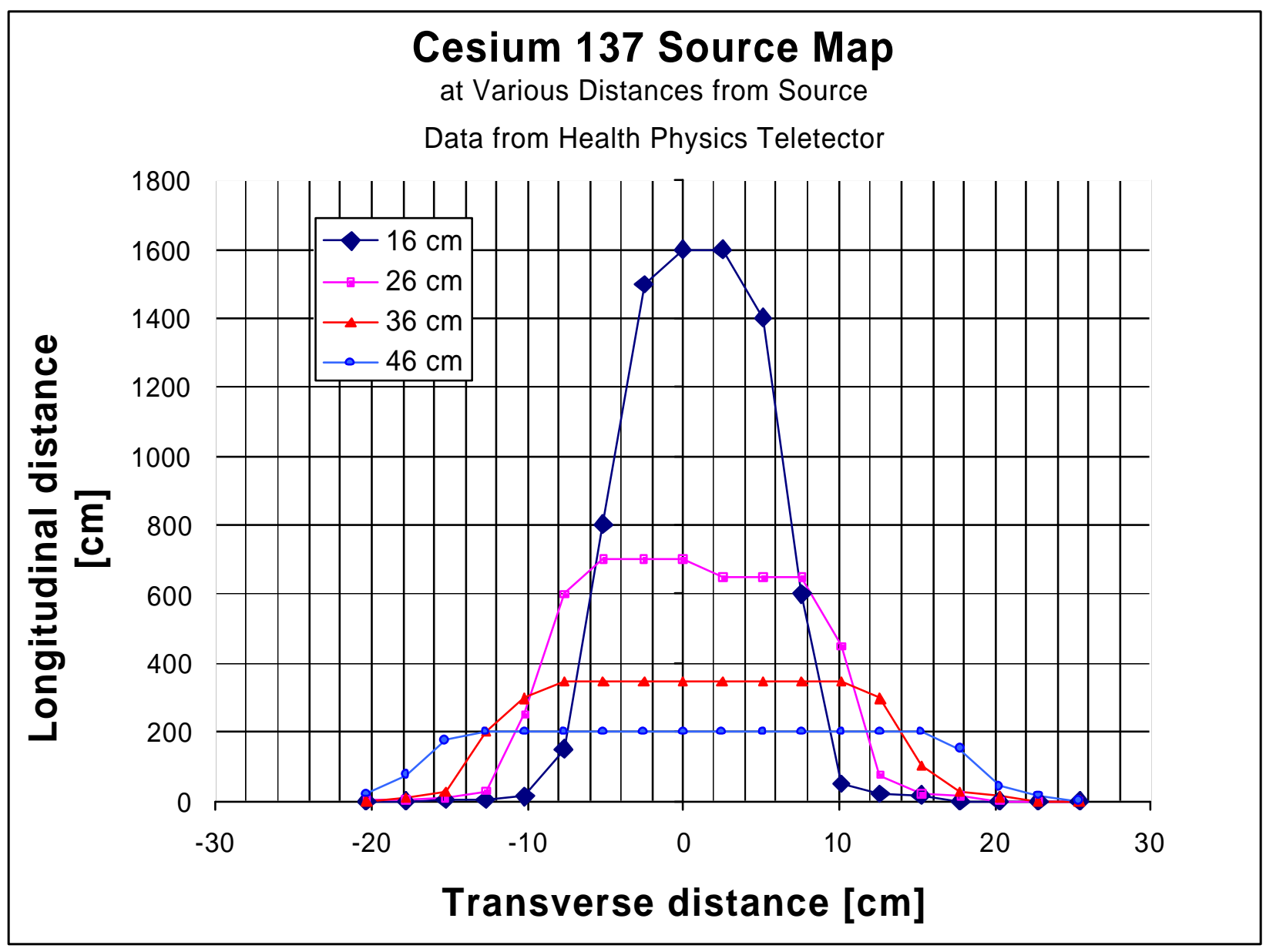

Figure 1. Plot of Cesium 137 source transverse distribution.

The on-axis data was replotted along with an inverse-square fall-off. The agreement was quite acceptable.. The results are shown in Figure 2, below. 


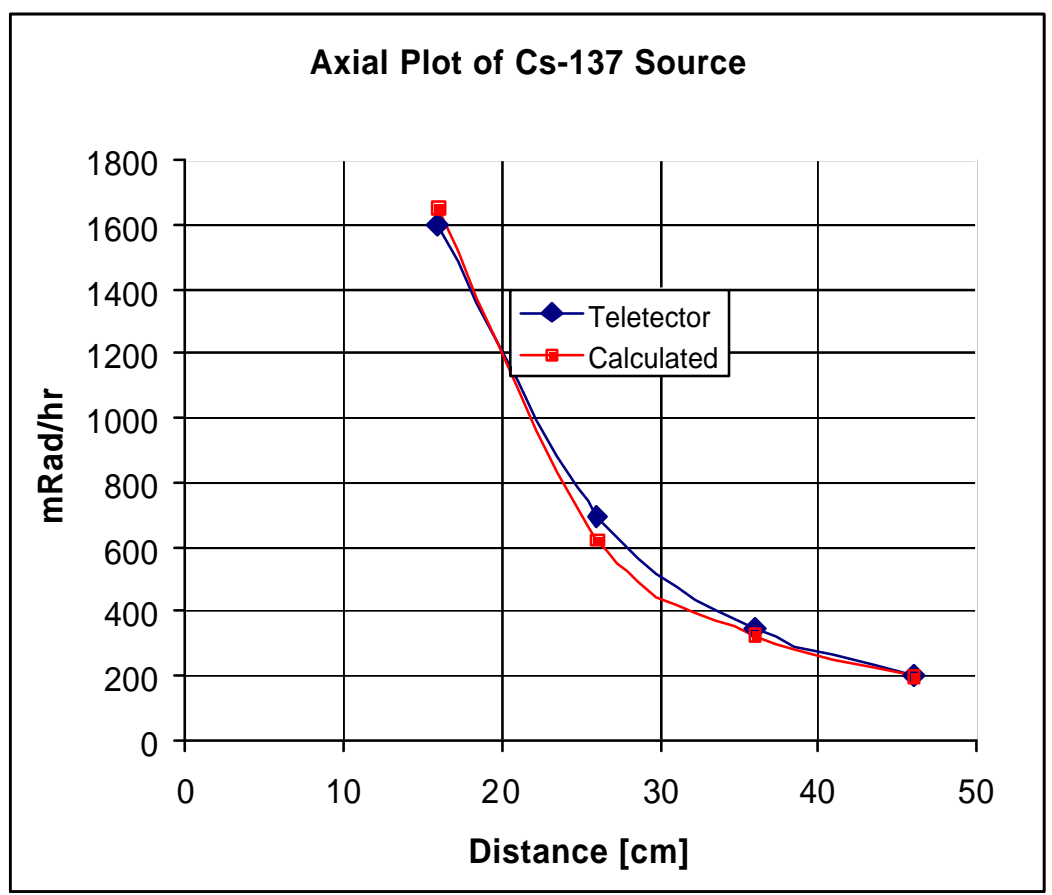

Figure 2. Fall off of axial source intensity versus distance with calculated values.

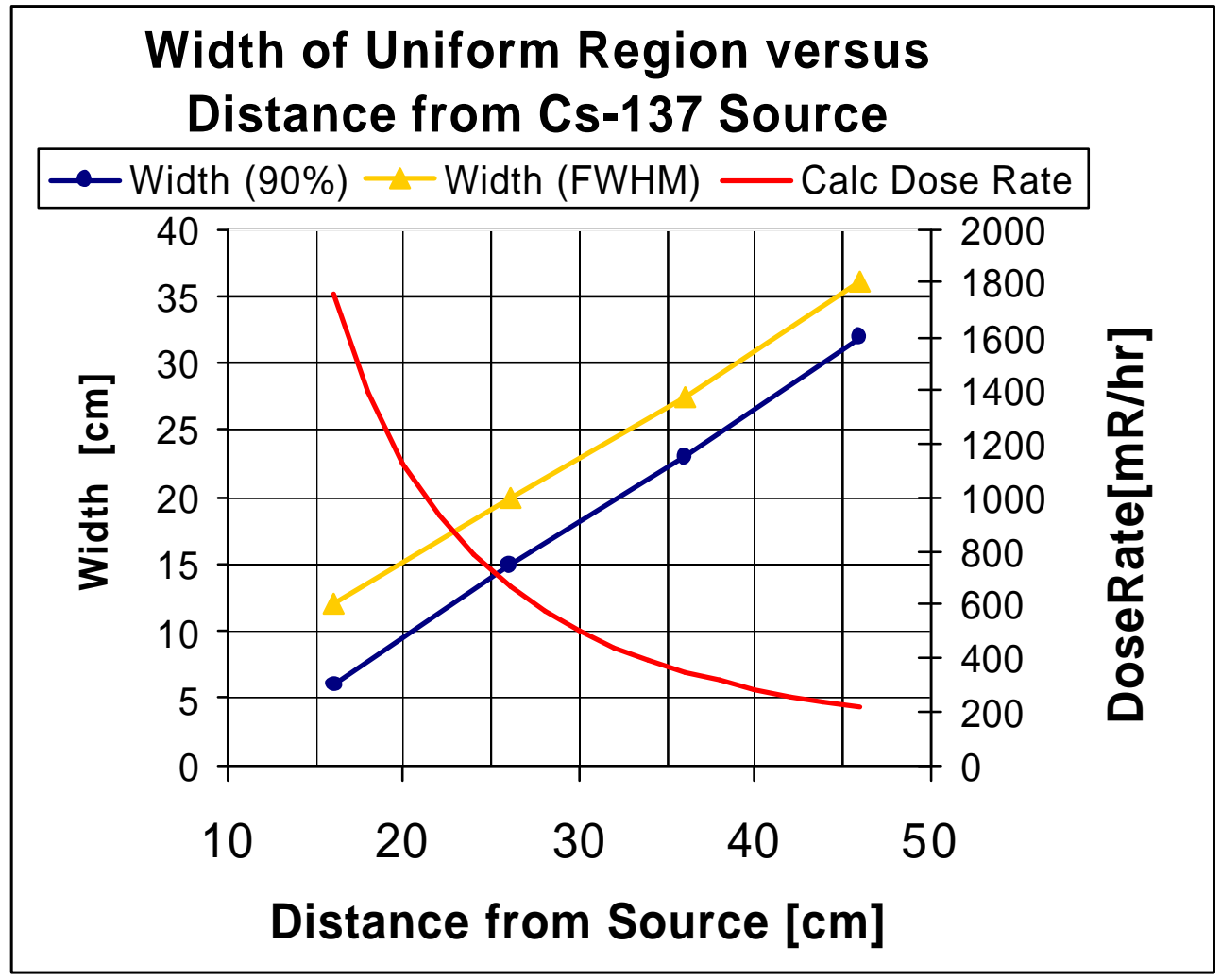

Figure 3. Width of uniform coverage. 
Whereas the coverage at $20 \mathrm{~cm}$ was sufficient for the RHIC BLMs, the location must be moved to $30 \mathrm{~cm}$ to accommodate the SNS detectors. At the new location the dose rate will be a little less than half that at $20 \mathrm{~cm}$. Thirty $\mathrm{cm}$ was chosen as the new location.

\section{The Electronics}

The original electronics were based on a low leakage gated integrator developed for use in the AGS. Since the ion chamber acts like a true current source the integrated signal was a straight line. The data was taken on a Hewlett Packard digital scope triggered at a 2 $\mathrm{Hz}$ rate. The same trigger was used to resent the integrator. The digitized scope trace was acquired using LabView. The program took the data and fitted a straight line using a least squares fitting routine. The slope of the line is the current. To further smooth the data the measurement was repeated for a total of 8 readings which were then averaged. The Lab view program then incremented the high voltage and after a sufficient settling delay the measurement was repeated. This procedure was executed twice: once with the source shielded in its housing so that the baseline of the electronics and high voltage leakage of the detector could be measured, and again with the source in position.

\section{Changes to the Electronics}

The new setup uses a Kiethley model 248 HV power supply and a Kiethley model 6517 A Electrometer. The LabView program (VI) was rewritten to control these units while presenting the same operator interface and providing the same data output. The resulting scans showed considerably less sensitivity to noise when the data was averaged over 100 measurements. A block diagram of the test facility is shown in Figure 4.

The Ground Break shown interrupts the shield of the signal cable. This is necessary because of a $100 \mathrm{MOhm}$ resistor shunting the signal in the RHIC and SNS BLM endcaps. The $100 \mathrm{MOhm}$ resistor prevents the cable from charging to the full $\mathrm{HV}$ power supply voltage in the event that it is disconnected from the signal electronics while the HV power supply is on. However, this resistor also presents a parallel path for the signal current. In the past, (and for the actual AFE electronics) the input impedance of the test stand electronics was at least 5 orders of magnitude less so the small diverted current was unmeasurable. However the dynamic input impedance of the Kiethley 6517A appears to be about $10 \mathrm{MOhms}$ and cannot be ignored. Since the BLM ion chamber is a true current source breaking the ground connection does not affect the signal current from the detector but does prevent current from flowing through the $100 \mathrm{MOhm}$ resistor. 


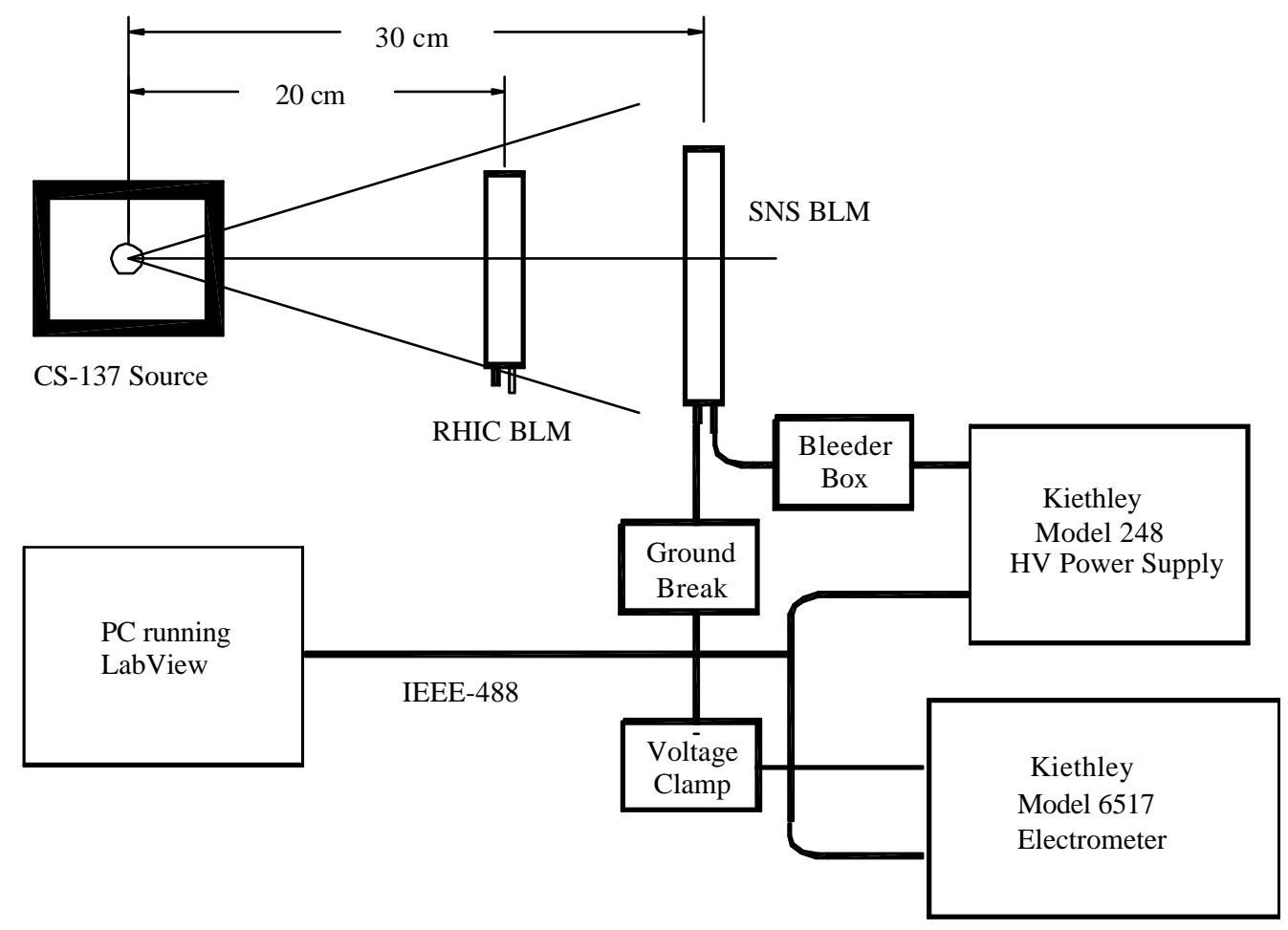

Figure 4. Block diagram of the BNL BLM Test Facility

Figure 5 shows a typical calibration run. As expected the signal level is lower due to the SNS chamber being located further from the source.

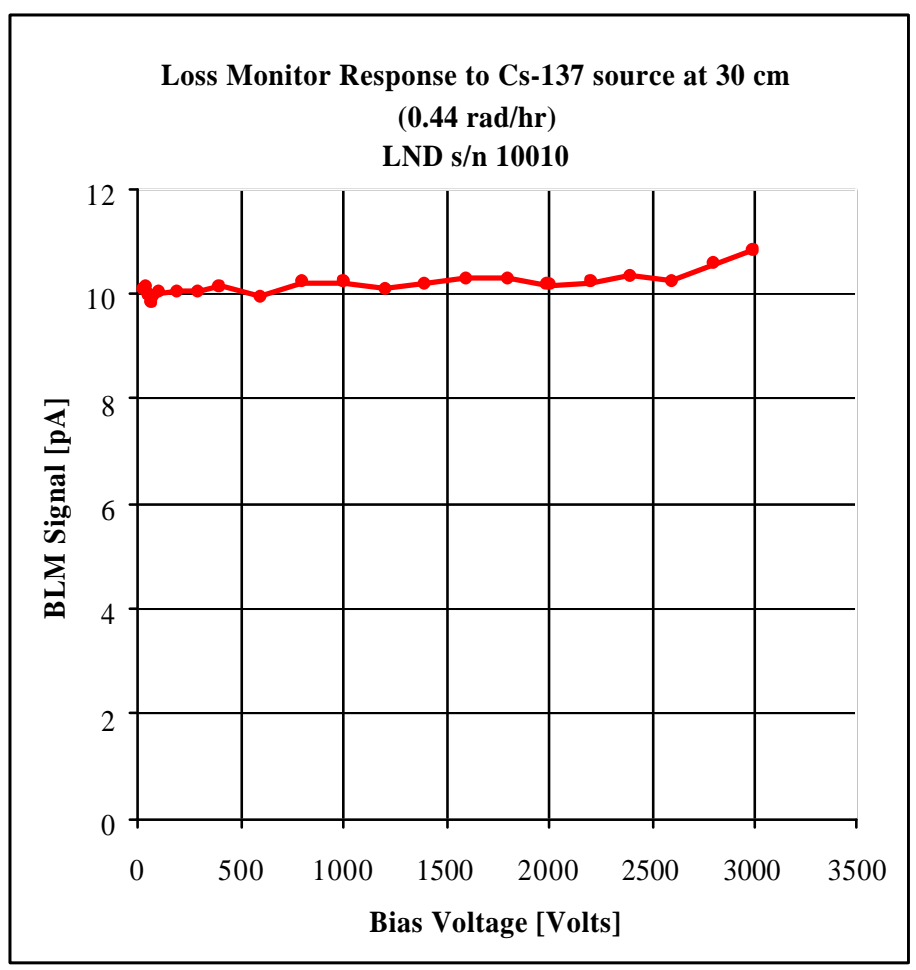

Figure 5. Calibration of an SNS BLM. 


\section{Acknowledgements}

Paul Ziminski has been responsible for testing all of the RHIC BLMs and provided valuable input in the design and construction of the original and new BLM Test Stands.

\footnotetext{
${ }^{1}$ R. E. Shafer, et al., "The Tevatron Beam Position and Beam Loss Monitoring Systems", Proc. $12^{\text {th }}$ Int'l Conf on High Energy Accel., FNAL (1983) p609

2 Troy-Ionics, Kenville, NJ

3 R. Witkover, D. Ga ssner, "Design and Testing of the New Ion Chamber Loss Monitor for SNS" Proc of 2003 PAC, to be published
} 